\title{
Comment on the mean-square deviation formula for autoionizing states
}

\author{
Cleanthes A. Nicolaides \\ Research Institute for Physics, 10405 Stockholm 50, Sweden \\ and Theoretical Chemistry Institute, National Hellenic Research Foundation (E.I.E.), Athens 501/1, Greece \\ Donald R. Beck \\ Belfer Graduate School of Science, Yeshiva University, New York, New York 10033 \\ and Theoretical Chemistry Institute, National Hellenic Research Foundation (E.I.E.), Athens, 501/1, Greece \\ (Received 19 July 1976)
}

We offer a derivation of the mean-square deviation formula, recently employed in variational calculations of autoionizing states, from a time-dependent point of view.

In recent paper $\mathrm{s}^{1-4}$ the formula

$$
\delta \Delta=\delta\left\langle(H-\langle H\rangle)^{2}\right\rangle=0
$$

has formed the basis for variational calculations of autoionizing states and energies. We would like to offer a conceptually simple and meaningful derivation of the above formula.

In Refs. 5 and 6 we adopted a time-dependent point of view for understanding and computing the properties of autoionizing states. Further work along these lines has been completed recently. ${ }^{7-9}$ The autoionizing states are treated as nonstationary states satisfying

$$
\psi(t)=e^{-(i / \hbar) H t}\left|\psi_{0}\right\rangle
$$

with

$$
\begin{aligned}
& \left|\psi_{0}\right\rangle \equiv|\psi(t=0)\rangle, \\
& H_{0}\left|\psi_{0}\right\rangle \equiv(H-V)\left|\psi_{0}\right\rangle=E_{0}\left|\psi_{0}\right\rangle .
\end{aligned}
$$

Physically the state $\left|\psi_{0}\right\rangle$ is defined uniquely. It is the initial, localized state which describes all the interactions at $t=0$. Mathematically, it can be represented by an exact square-integrable wave function satisfying the Schrödinger differential equation with asymptotically vanishing boundary conditions. It is related to the Gamow complex wave functions with complex eigenvalues in the second Riemann sheet, by analytic continuation. ${ }^{9}$

The very notion of a decaying state implies the following limit:

$$
|\psi(t)\rangle \rightarrow\left|\psi_{0}\right\rangle, \text { as } t \rightarrow 0 \text {. }
$$

For a stationary state, the expression

$$
\Delta=\left\langle(H-\langle H\rangle)^{2}\right\rangle=0 .
$$

Thus, one intuitively would expect that, when the limit of Eq. (5) is taken,

$$
\Delta\left(\psi_{0}\right) \equiv\left\langle\psi_{0}\left|(H-\langle H\rangle)^{2}\right| \psi_{0}\right\rangle=\min .
$$

This can be seen from the following considera- tion: Let

$$
u(t) \equiv \psi(t)-\psi_{0}(t),
$$

where $\psi(t)$ satisfies Eq. (2) and

$$
\psi_{0}(t)=e^{-(i / \hbar) H_{0} t}\left|\psi_{0}\right\rangle .
$$

We now consider an infinitesimal unitary transformation such that

$$
\left\|\lim _{t \rightarrow 0} u(t)\right\|=\min
$$

or

$$
\| \lim _{t \rightarrow 0}\left(e^{-i / \hbar H t}-e^{-i / \hbar H_{0} t}\right)\left|\psi_{0}\right\rangle \|=\min .
$$

The physically meaningful and mathematically correct limit is then obtained by allowing

$$
\begin{gathered}
e^{ \pm(i / \hbar) H t} \underset{t \rightarrow 0}{\sim} 1 \pm(i / \hbar) H t, \\
e^{ \pm(i / \hbar) H}{ }_{t \rightarrow 0} \underset{t}{\sim} 1 \pm(i / \hbar) H_{0} t,
\end{gathered}
$$

so that $H$ and $H_{0}$ are, as required, the generators of the canonical transformation.

Substitution of Eqs. (12) into Eq. (11) yields the desired relationship, Eq. (7), and from it

$$
\delta \Delta\left(\psi_{0}\right)=\delta\left(\left\langle\psi_{0}\left|H^{2}\right| \psi_{0}\right\rangle-\left\langle\psi_{0}|H| \psi_{0}\right\rangle^{2}\right)=0 .
$$

Equation (13) employs square-integrable wave functions.

Applications of this formula to He autoionizing states and to the Stark effect from different points of view have been presented in Refs. 1, 3, and 4 . Also in Ref. 3, a derivation of Eq. (13) is presented by requiring that the variation of the decay function with respect to $\psi_{0}$ is zero [Eq. (4) of Ref. 3].

Note added in proof. In Ref. 10, a new variational principle is presented which employs complex functions and matrix elements of $H^{2}$ and yields both 
the energy and the width of resonances. In the limiting case where the complex parameters or coordinates of the basis functions become real, this principle reduces to Eq. (13).
C.A.N.gratefully acknowledges a NORDITA fellowship. A useful exchange of ideas with Dr. Brändas on the properties of autoionizing states is also acknowledged with pleasure.
${ }^{1}$ P. Froelich and E. Brändas, Phys. Rev. A 12,1 (1975).

${ }^{2}$ E. Brändas and P. Froelich, Int. J. Quantum. Chem.

S9, 457 (1975).

${ }^{3}$ I. Oksüz, Phys. Rev. A 13, 1507 (1976).

${ }^{4}$ F. H. Read, Chem. Phys. Lett. 12, 549 (1972).

${ }^{5}$ C. A. Nicolaides, Phys. Rev. A $\underline{6}, 2078(1972)$.

${ }^{6}$ C. A. Nicolaides, Nucl. Inst. Methods 110, 231 (1973).

${ }^{7}$ C. A. Nicolaides and D. R. Beck, Beamfoil Spectros- copy, edited by D. Pegg and I. A. Sellin (Plenum, New York, 1976), Vol. 1, p. 77.

${ }^{8}$ C. A. Nicolaides and D. R. Beck, J. Chem. Phys. (to be published).

${ }^{9}$ C. A. Nicolaides and D. R. Beck (unpublished).

${ }^{10} \mathrm{C}$. A. Nicolaides and D. R. Beck, Phys. Lett. A (to be published). 